\title{
Diez obviedades con motivo de la concesión del Premio Antonio Gallego
}

\author{
Albert Oriol Bosch \\ Fundación Educación Médica
}

Con motivo de la concesión del Premio Antonio Gallego quisiera exponer 10 obviedades, 3 de ellas circunstanciales y 7 educativas.

\section{Obviedades circunstanciales}

En primer lugar reconocer el acierto de la Sociedad Española de Educación Médica en instaurar un premio en honor a su fundador y primer presidente, Prof. Antonio Gallego Fernández. En segundo lugar agradecer la concesión confiando en que en el futuro se honren biografías de mayor relevancia y finalmente agradecer a todos los que han ayudado (hecho posible) aquellos méritos que el jurado haya sabido encontrar en mi persona (ver addenda)

\section{Obviedades educativas}

1. En el proceso educativo el protagonista es siempre el que aprende. El que enseña no es más que un recurso de ayuda.

2. La importancia del que enseña reside tanto en su rol modelizador como en su experteza de contenidos y procesos.

3. La educación médica no es una finalidad en si misma y debe perseguir un fin (educación orientada a resultado).

4. Deben usarse variedad de metodologías educativas ajustadas en cada caso a los objetivos perseguidos. Para dominar las diversas metodologías no hay otro mecanismo que practicar con ellas.

5. Por razones éticas y prácticas la educación médica deberá utilizar en mayor medida las simulaciones como instrumento educativo.

6. La evaluación es parte esencial del proceso educativo y debe incorporarse ya en la fase del diseño de las actividades docentes.

7. La metodología evaluativa debe ser robusta y estar alineada con los objetivos de aprendizaje.

\section{Addenda}

Agradecimientos profesionales rememorados a vuelapluma y ordenados por periodos:

Los modelos familiares:

José y Antonio Oriol Anguera cuyas vidas fueron destruidas por una tragedia colectiva y que supie- ron rehacerla y una familia tribal con valores ejemplarizantes.

Modelos y grandes maestros:

Profesores Artur Jores y Klaus Dieter Voigt de la $2^{\text {a }}$ Clínica Médica Universitaria del Hospital Eppendorf de Hamburgo.

Profesores Leo T. Samuels y Kristen B. Eik-nes del Departamento de Bioquímica de la Universidad de Utah (Salt Lake City)

Profesores Gregory Goodwin Pincus y Elijah Romanoff de la Worcester Foundation for Experimental Biology (Shrewsbury, Mass)

Profesor Antonio Gallego Fernandez

Colaboradores, compañeros y discípulos:

Josep Cabré Piera (†), Eduardo Basilio, Francesc Doménech, Alfredo Marqués, Jordi Setoain, Arturo Sarró, Toni Martí, Pere Pujol, etc. compañeros de estudios, deporte y aventuras juveniles.

Apostolakis (Tesalónica), Zubiate (Lima), Parada (Concepción), Paleschi (Nápoles), Frahm (Hamburgo), Tamm (Hamburgo) y otros que me ayudaron durante mi estancia en Hamburgo.

Domínguez (México), Nishizawa (Toronto), Aakvaag (Oslo), López (Manila), van der Molen (Rotterdam), Brownie (Glasgow), Hall (Sidney), Billiar (Nebraska), Rosner (Buenos Aires), y otros miembros de la legión de "esteroidólogos" y "reproductivistas".

Carlos Belmonte, Margarita Barón, Luis Clos, Antonio Torralba, Antonio de la Fuente del equipo del Profesor Antonio Gallego que me acogieron con benevolencia y cariño.

Los Profesores Enrique Aguilar y Juan Jorda ( $†$ ), los hermanos Durán, Ramiro Diez Lobato, Alicia Tejero y todos los alumnos de la Complutense que sufrieron mis primeras experiencias docentes y ayudaron en las tareas de experimentación en el laboratorio.

El Profesor Pedro Mestres y los doctores Carlos Soler Durall con los que pusimos en marcha la Facultad de Medicina de la Universidad Autónoma de Barcelona y los numerosos colegas del Hospital de San Pablo sin cuya colaboración no hubiera sido posible hacerlo: Gonçal Lloveras ( $\dagger$ ), Francesc Morer $(\dagger)$, Joaquim Borrull $(\dagger)$, Joaquim Bonal $(\dagger)$, Antoni Oriol, Ramón Trías, Grau, Barraquer, Rutllant, 
Raventós, y tantos otros. Los alumnos de las tres primeras promociones de la Autónoma de Barcelona que fueron banco de pruebas y estimulo,

Los Profesores Jesús Tresguerres y Javier Rodríguez, Esteban Mancheño, Rafael García Robles ( $\dagger$ ), Javier Bagazgoitia, Antonio Gómez Pan, Luis Miguel Ruilope, Basilio Moreno y muchos otros que hicieron de la Cátedra de Endocrinología Experimental un lugar creativo y divertido.

Los distinguidos miembros del Claustro de la Facultad de Medicina de la Universidad Complutense y muy especialmente los Profesores Pedro Laín Entralgo (†), Amador Schuller, Rosendo Poch, Diego Gracia, Pedro Zarco, Rafael Lozano y los compañeros del Hospital de San Carlos y del Hospital 12 de Octubre con los que intentamos innovar la docencia y las estructuras institucionales.

Al Muy Honorable Jordi Pujol y al Honorable Josep Laporte $(\dagger)$, de cuyo liderazgo disfruté largos años.

A José $\mathrm{M}^{\mathrm{a}}$ Martínez Carretero por su capacidad en desplegar el Área de Evaluación de las Competencias Clínicas y su fidelidad, a Josep Roma y Maria Nolla, cuya capacidad analítica y crítica contribuyó al desarrollo institucional del IES así como a sus técnicos y personal sin los que mi vida no habría podido ser como ha sido.

A Randol Barrer de la Universidad Johns Hopkins y Ronald Epstein de la Universidad de Rochester que nos enriquecieron durante su estan- cia sabática en el IES con nuevas perspectivas y nos transfirieron sus experiencias. Asimismo Miriam Friedman Ben-David ( $\dagger$ ), y Carlos Brailovsky (Universidad de Québec) cuyo apoyo fue más allá de lo estrictamente técnico.

Capítulo especial para Milagros Garcia Barbero, Andrej Wojtzak y Charles Boelen que desde sus posiciones en la WHO pero también desde fuera nos ofrecieron la ayuda de su experiencia, conocimientos y afecto.

A una pléyade de personas vinculadas a instituciones relevantes que me han ofrecido su ayuda a lo largo de los años tales cómo: Asociación Española contra el Cáncer, Comisión Fullbright, Fundación March, Fundación Marqués de Urquijo, Educational Comissionn for Foreign Medical Graduates, National Board for Medical Examiners, Network for Community Oriented Educational Institution-TUFH, Institute for Internacional Medical Education, Academia de Ciencias Médiques de Catalunya i de Balears, IVIMEDS, AMEE, AMDE, WFME.

A un numeroso colectivo de colegas y amigos del sector sanitario con los que he compartido las tareas de intentar resolver problemas y de los que me es difícil trazar la línea que separa el afecto, las preocupaciones y responsabilidades compartidas, de las enseñanzas que me ofrecieron y que por pudor y prudencia no menciono.

Barcelona 11 de Septiembre de 2005 